\title{
Temporal Development of Ion Beam Mean Charge State in Pulsed Vacuum Arc Ion Sources
}

\author{
E.M. Oks $^{1,2 *}$, G.Yu. Yushkov ${ }^{2}$ and A. Anders ${ }^{3}$ \\ ${ }^{1}$ State University of Control Systems and Radioelectronics, Tomsk, 634050, Russia \\ Fax:+7-3822-414859,E-mail address:oks@fet.tusur.ru \\ ${ }^{2}$ High Current Electronics Institute, Russian Academy of Sciences, Tomsk 634055, Russia \\ Fax: +7-3822-492410, E-mail address: gyushkov@opee.hcei.tsc.ru \\ ${ }^{3}$ Lawrence Berkeley National Laboratory, Berkeley, California 94720, USA \\ Fax: +1-510-4864374,E-mail address: aanders@lbl.gov
}

\begin{abstract}
Vacuum arc ion sources, commonly also known as "Mevva” ion sources, are used to generate intense pulsed metal ion beams. It is known that the mean charge state of the ion beam lies between 1 and 4, depending on cathode material, arc current, arc pulse duration, presence or absence of magnetic field at the cathode, as well background gas pressure. A characteristic of the vacuum arc ion beam is a significant decrease in ion charge state throughout the pulse. This decrease can be observed up to a few milliseconds, until a "noisy" steady-state value is established. Since the extraction voltage is constant, a decrease in the ion charge state has a proportional impact on the average ion beam energy. This paper presents results of detailed investigations of the influence of arc parameters on the temporal development of the ion beam mean charge state for a wide range of cathode materials. It is shown that for fixed pulse duration, the charge state decrease can be reduced by lower arc current, higher pulse repetition rate, and reduction of the distance between cathode and extraction region. The latter effect may be associated with charge exchange processes in the discharge plasma.
\end{abstract}




\section{INTRODUCTION}

A vacuum arc based ion source is known as an attractive method to produce high current beams of metal ions. This kind of ion source has been used for ion implantation in material surface modification research, and for particle accelerator injection for fundamental nuclear physics research, and also for other fundamental and applied purposes. Beams have been produced from over 50 of the solid metals of the Periodic Table, with ion energy of about $100 \mathrm{keV}$ and with beam current up to $1 \mathrm{~A}$. The source is usually repetitively pulsed with pulse length up to several milliseconds and pulse repetition rate from 0.1 to 50 pps. In general the ions have low but multiply-ionized charge states. The mean charge state lies between 1+ and 4+, depending on cathode material, arc current, arc pulse duration, presence or absence of magnetic field at the cathode, as well background gas pressure. The charge states can be increased in a number of different ways. Detailed reviews of recent development of the vacuum arc ion sources and its application have been given elsewhere $[1,2]$.

For vacuum arc ion sources it was observed that a significant decrease of ion beam charge states occurs throughout the pulse. This decrease can be observed up to a few milliseconds [3] and it has a proportional impact on the average ion beam energy. The ion beam mean charge state is determined by the fraction of differently charged ions in the vacuum arc plasma. The physical aspects of the reduction of ion charge states in vacuum arc plasma were subject of our detailed investigations [4,5], which have confirmed that the reduction of ion mean charge after arc initiation is consistent with the evolution of neutrals in the volume through which the metal plasma flows. This paper considers the reduction of 
the ion mean charge state throughout the vacuum arc pulse from the point of view of its influence on the parameters of a vacuum arc ion source.

\section{EXPERIMENTAL SET-UP}

A conventional version of the vacuum arc metal ion source "Mevva-V" [6] with up to 18 mounted cathodes for arc operation was used in experiments that were done at Berkeley Lab. The ion source (Fig.1) consists of a plasma source, and three grid of multiaperture system of the acceleration-deceleration type. Ion beam mass/charge state distributions were measured by a time-of-flight (TOF) spectrometer [7]. The TOF instrument has a drift length of $1.03 \mathrm{~m}$ and the detector was a magnetically suppressed Faraday cup. Cathode spots could only burn on the cathode rod's front surface, with the metal plasma expanding through an annular anode of $1 / 2$ inch $(12.5 \mathrm{~mm})$ inner diameter into a plasma expansion chamber that had about $10 \mathrm{~cm}$ diameter and $10 \mathrm{~cm}$ length.

Each arc discharge of 100 - 400 A current had duration of $300 \mu$ s, determined by the pulse-forming network, at a repetition rate from 0.1 to 10 pulses per second (pps). The plasma streams from the cathode spots towards the extraction grid system. The suppressor grid voltage was $-2 \mathrm{kV}$ and the extraction voltage was $40 \mathrm{kV}$. Different metals (Al, $\mathrm{Mg}, \mathrm{Cu}$, Pb, Bi, Mo) were used as cathode in experiments. Molybdenum was use as a base cathode material mainly because its ions charge states include ion species from $1+$ to $5+$ and with so wide range of different charged states it was rather convenient to recognize the changing of charge state during the arc current pulse. The evolution of the ion beam charge state distribution was measured at 10 different times after arc triggering from $25 \mu$ s to $250 \mu \mathrm{s}$ 
with steps of $10 \mu \mathrm{s}$ or $25 \mu \mathrm{s}$. All measurements were made in average mode of the Tektronix TDS 744 digital oscilloscope. The number of averaging pulses depended on the pulse repetition rate and was selected $N=10$ for $f=0.1$ pps, 25 for 1 pps and 100 for 10 pps. The arc voltage was measured directly from cathode and anode electrode contacts by a fast 1:100 voltage divider.

In experiments with changing the distance between cathode and extraction grid (that was connected electrically to anode) the standard 18-cathode holder was removed and a movable, single cathode plasma source installed (“minigun” [8]). The cathode size was the same as with the 18-cathode source. For all experiments, the background pressure was about $10^{-6}$ Torr.

\section{RESULTS}

The absolute value of the discharge voltage $U_{d}$ was affected by arc current, pulse repetition rate and cathode - grid distance. For the central part of the arc pulse, i.e. from 25 $\mu$ s to $250 \mu$ s, the shapes of arc current and discharge voltage were practically constant.

Despite constant discharge parameters, the ion beam mean charge state showed essential fall during each arc pulse (Fig. 2). The experimental data of the decrease of $<Q_{i}>$ can be fitted with a first-order exponential function of the form

$$
\bar{Q}(t)=A \exp (-t / \tau)+\bar{Q}_{s s}
$$

where $A$ is a factor that describes the relevance of the decay, $\tau$ is the characteristic decay

time, and $\bar{Q}_{s s}$ is the steady-state average charge state number which is approached for $t$ 
$>>\tau$. The strongest decay is seen with Bi could be explained by the special thermal properties of this materials [4].

The discharge voltage showed a noticeable rise with increase of arc current but again the shape of $U_{d}(t)$ remained constant during the arc pulse (Fig.3). Higher discharge voltage together with higher arc current need to be considered because of their product gives an essential enhancement of the power dissipation in the discharge area. It implies higher electron temperature in the vacuum arc plasma and thus higher ion charge states. That, however, turned out to be true only at the beginning of the pulse (Fig.4). One can see from Fig.4 that an increase of arc current leads to a greater decrease of the ion beam mean charge state. So, at the end of the arc current pulse, the influence of the discharge current has actually changed into the opposite. The smaller arc current the higher ion charge state. Such influence of arc current clearly indicates the simultaneous existence of two contrary processes. The first one provides a higher ionization rate and the second a decrease ion mean charge state that can due, for example, to the production of neutral gas followed by charge exchange in the discharge area.

It is known that, at low arc pulse repetition rates, the plasma contains large amounts of non-metal ion species such as hydrogen and oxygen, and that the ion charge states are reduced compared to high-repetition rate operation [9]. The influence of the repetition rate on the temporal development of the ion beam mean charge state (Fig.5) could be consider as further evidence for the important role of neutrals. At nearly the same discharge voltage, higher repetition rate lead to a little lower reduction of the ion beam mean charge state at the end of the pulse. 
The dependence of the charge state reduction on the distance between cathode and extraction grid (i.e., the path length ions have to travel before they are extracted and analyzed) is presented in Fig. 6. For this measurement, the cathode assembly was mounted on a movable feedthrough. With the same arc current value, changing the distance from 1 $\mathrm{cm}$ to $8 \mathrm{~cm}$ increased discharge voltage from 37 to $40 \mathrm{~V}$. But one can clearly see from Fig.6 that the greater the distance the lower the mean ion charge state.

\section{CONCLUSION}

For all investigated vacuum arc ion sources cathode materials, the arc current, the pulse repetition rate, and the length of cathode-anode distance have an essential influence on the development of the ion beam mean charge state distribution. These results indicate the important role of neutrals (both metal and gas) for charge exchange processes in the discharge volume between cathode and anode. To obtain higher charge states during the whole pulse it is necessary to decrease the distance between cathode and ion extraction area as well as to use smaller arc current and higher pulse repetition rate.

\section{Acknowledgments}

The authors are grateful to the Mevva source’s “father” Dr. Ian Brown for helpful discussions. This work was supported by the U.S. Department of Energy, Office of Nonproliferation and International Security, Initiatives for Proliferation Prevention, Project No. IPP-LBNL-T2-196, under Contract No. DE-AC02-05CH11231 with the Lawrence Berkeley National Laboratory. 


\section{References}

1. The Physics and Technology of Ion Sources. Editor Dr. I. Brown. Second Revised and Extended Edition Wiley -VCH, 2004, Chapter 13 “Vacuum arc ion sources”.

2. Ian Brown and Efim Oks, IEEE Trans. Plasma Sci., 33, 1931, (2005).

3. Reich H.; Spadtke P.; Oks E.M., 71, 707, (2000).

4. A. Anders and G. Y. Yushkov, Appl. Phys. Lett. accepted for publication (2007).

5. A. Anders E.M., Oks and G. Y. Yushkov, J. Appl. Phys. accepted for publication (2007).

6. I. G. Brown, Rev. Sci. Instrum. 65, 3061, (1994).

7. Gushenets VI, Nikolaev AG, Oks EM, et al., Rev. Sci. Instrum., 77, 063301 (2006).

8. R. A. MacGill, M. R. Dickinson, A. Anders, O. R. Monteiro, and I. G. Brown, Rev. Sci. Instrum. 69, 801, (1998).

9. G. Y. Yushkov and A. Anders, IEEE Trans. Plasma Sci. 26, 220, (1998). 


\section{Figure Captions}

Fig. 1. Schematic of the LBNL Mevva-V source used in experiments.

Fig. 2. Ion beam mean charge state number for different cathode materials as a function of time after arc ignition with the arc current $300 \mathrm{~A}$ and pulse repetition rate of $7 \mathrm{pps}$.

Fig. 3. Arc voltage pulse shape for repetition rate $1 \mathrm{pps}$ for Mo cathode with the arc current as a parameter.

Fig. 4. Reduction of the ion beam mean charge state for Mo cathode with the arc current as a parameters. Repetition rate 1 pps

Fig. 5. Ion beam mean charge state number for different cathode materials as a function of pulse repletion rate for Mo cathode with the arc current $400 \mathrm{~A}$.

Fig. 6. Mean ion charge state number for Mo cathode (arc current 400A, 1 pps) as a function of time for different distances between cathode surface (location of ion production) and extraction grid (location of ion analysis). 


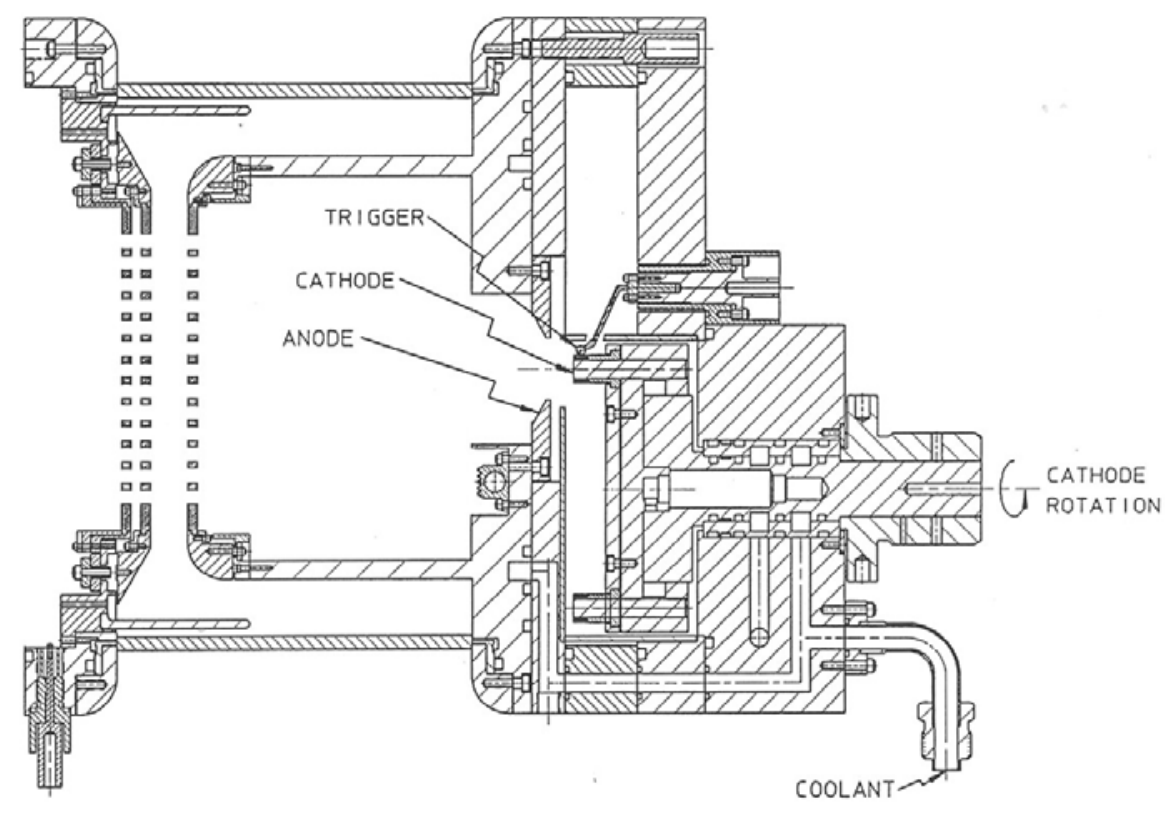

Fig. 1. E.M. Oks, G.Yu. Yushkov and A. Anders

"Temporal Development of Ion Beam Mean Charge State in Pulsed" 


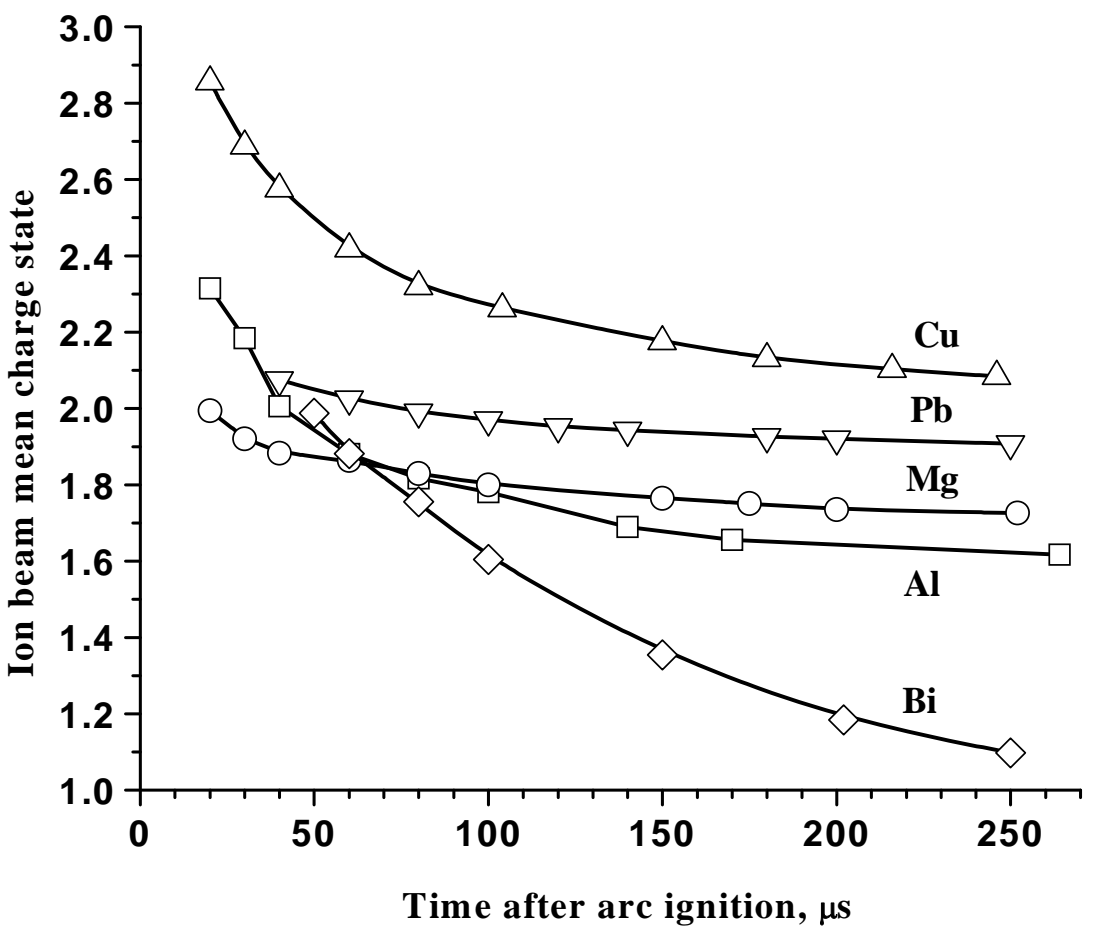

Fig. 2. E.M. Oks, G.Yu. Yushkov and A. Anders

“Temporal Development of Ion Beam Mean Charge State in Pulsed” 


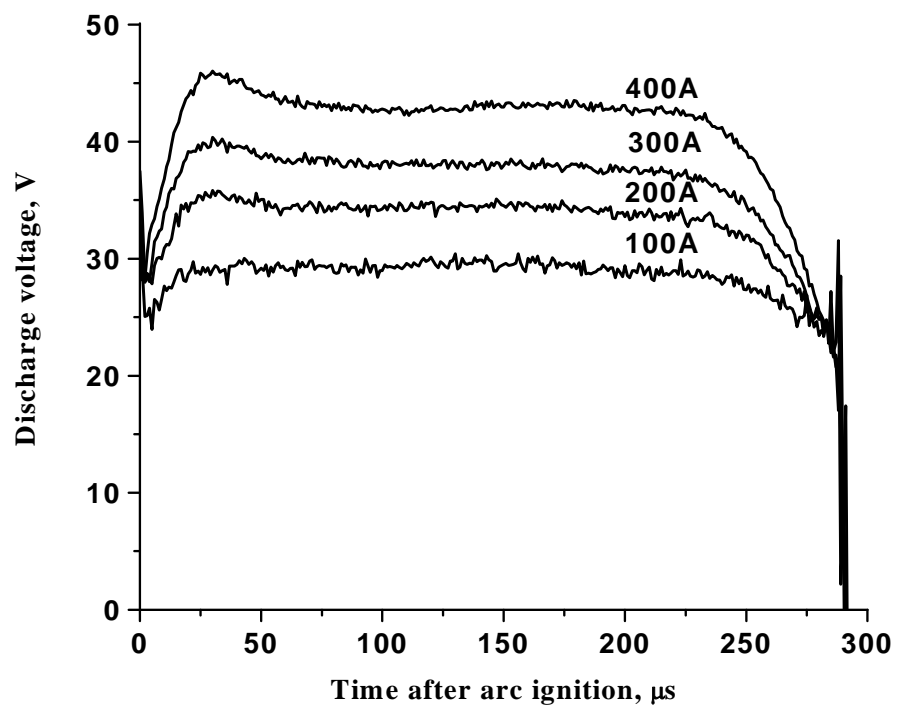

Fig. 3. E.M. Oks, G.Yu. Yushkov and A. Anders

"Temporal Development of Ion Beam Mean Charge State in Pulsed" 


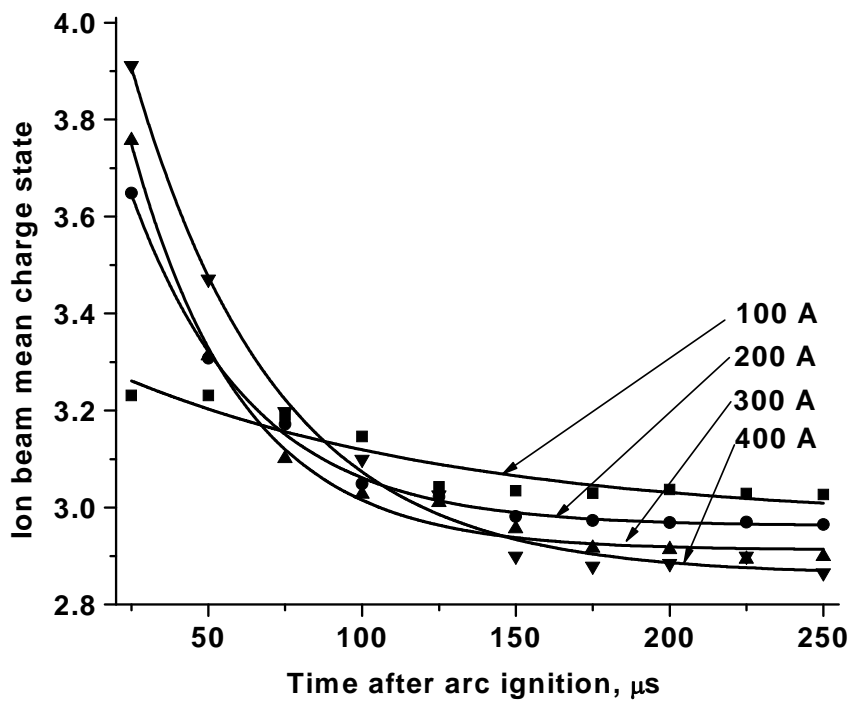

Fig. 4. E.M. Oks, G.Yu. Yushkov and A. Anders

“Temporal Development of Ion Beam Mean Charge State in Pulsed" 


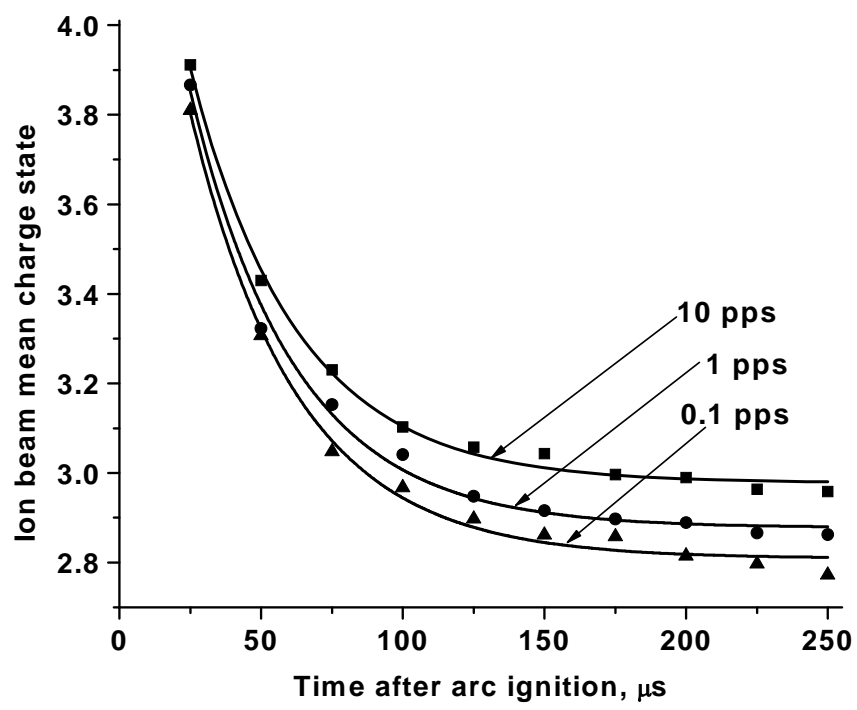

Fig. 5. E.M. Oks, G.Yu. Yushkov and A. Anders

"Temporal Development of Ion Beam Mean Charge State in Pulsed" 


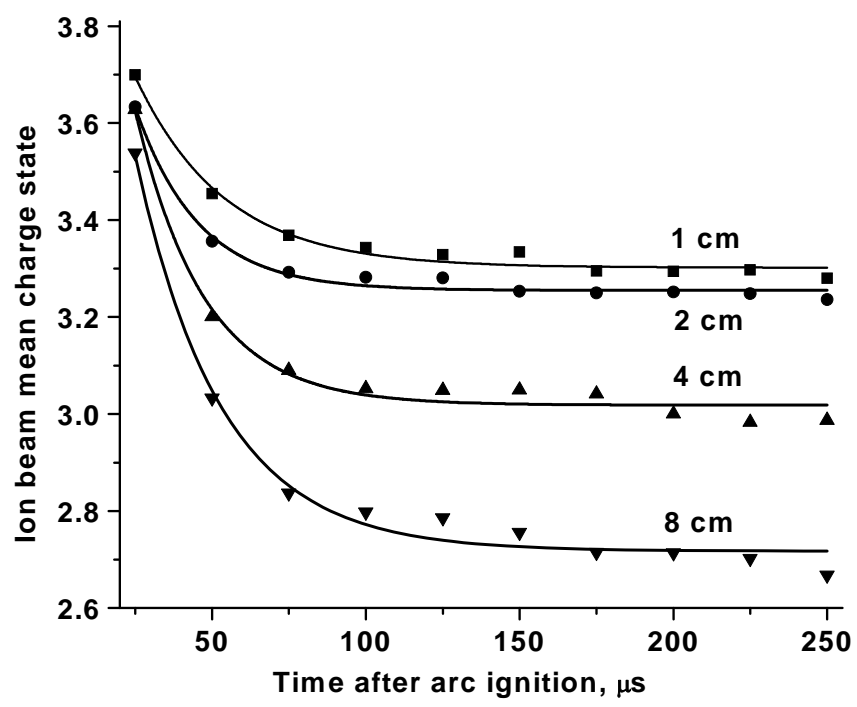

Fig. 6. E.M. Oks, G.Yu. Yushkov and A. Anders

"Temporal Development of Ion Beam Mean Charge State in Pulsed" 